\title{
A new eclipsing binary system with a pulsating component detected by CoRoT*
}

\author{
K. Sokolovsky ${ }^{1,2}$, C. Maceroni ${ }^{3}$, M. Hareter ${ }^{4}$, C. Damiani ${ }^{3}$, L. Balaguer-Núñez ${ }^{5}$, \\ and I. Ribas ${ }^{6}$ \\ ${ }^{1}$ Max-Planck-Institute für Radioastronomie, Auf dem Hügel 69, D-53121 Bonn, \\ Germany \\ ${ }^{2}$ Astro Space Center of Lebedev Phys. Inst., Profsoyuznaya 84/32, 117997 Moscow, \\ Russia \\ ${ }^{3}$ INAF - Osservatorio Astronomico di Roma, via Frascati 33, Monteporzio C., Italy \\ ${ }^{4}$ Institut für Astronomie, Universität Wien, Türkenschanzstraße 17, 1180 Vienna, \\ Austria \\ ${ }^{5}$ Departament d'Astronomia i Meteorologia-ICC-IEEC, Universitat de Barcelona, \\ Av. Diagonal, 647, 08028 Barcelona, Spain \\ ${ }^{6}$ Institut de Ciències de I'Espai (CSIC-IEEC), Campus UAB, Facultat de Ciències, \\ Torre C5, parell, 2a pl., E-08193 Bellaterra, Spain
}

\begin{abstract}
We report the discovery of CoRoT $102980178\left(\alpha=06^{\mathrm{h}} 50^{\mathrm{m}} 12^{\mathrm{s}} \cdot 10, \delta=-02^{\circ} 41^{\prime}\right.$ $21^{\prime \prime} 8, \mathrm{~J} 2000$ ) an Algol-type eclipsing binary system with a pulsating component (oEA). It was identified using a publicly available 55 day long monochromatic light curve from the CoRoT initial run dataset (exoplanet field). Eleven consecutive 1.26 deep total primary and the equal number of 0.25 deep secondary eclipses (at phase 0.50) were observed. The following light elements for the primary eclipse were derived: $H J D_{\mathrm{Minl}}=2454139.0680+5^{\mathrm{d}} 0548 \times E$. The light curve modeling leads to a semidetached configuration with the photometric mass ratio $q=0.2$ and orbital inclination $i=85^{\circ}$. The out-of-eclipse light curve shows ellipsoidal variability and positive O'Connell effect as well as clear 0 . 01 pulsations with the dominating frequency of $2.75 \mathrm{c} / \mathrm{d}$. The pulsations disappear during the primary eclipses, which indicates the primary (more massive)

\footnotetext{
* The CoRoT space mission was developed and is operated by the French space agency CNES, with participation of ESA's RSSD and Science Programs, Austria, Belgium, Brazil, Germany and Spain.
} 
component to be the pulsating star. Careful frequency analysis reveals the second independent pulsation frequency of $0.21 \mathrm{c} / \mathrm{d}$ and numerous combinations of these frequencies with the binary orbital frequency and its harmonics. On the basis of the CoRoT light curve and ground-based multicolor photometry, we favor the classification of the pulsating component as a $\gamma$ Doradus type variable, however, the classification as an SPB star cannot be excluded.

Accepted: June 25, 2010

Individual Objects: CoRoT 102980178

\section{Introduction}

Pulsating stars in eclipsing binary systems are objects of considerable astrophysical interest. It is well known that in double-line binaries masses, radii and luminosities of the components can directly be determined allowing us to constrain pulsation models. Possible influence of one component of a binary system on pulsations of the other component (through mass transfer or tidal interaction) is an interesting and still largely unexplored topic. A case of tidal excitation of $\gamma$ Doradus type pulsations was reported by Handler et al. 2002 (see also references to some theoretical discussions therein). In binary systems with one oscillating component the change of radius can be directly observed during the pulsation cycle by determining periodic changes in the times of the first to fourth contact. Bíró \& Nuspl (2005) suggest that the pulsation mode can be identified by using the secondary component as a spatial filter. Altogether, the identification of pulsating stars of different types in eclipsing systems is important.

The combination of pulsations and eclipses is often found among the evolved stars like symbiotic binaries (e.g. Chochol \& Pribulla 2000) and cataclysmic variables (e.g. Araujo-Betancor et al. 2005). Four Type I or II Cepheids in eclipsing binaries have recently been identified (see Antipin et al. 2007 and references therein), the confirmation of one more possible object of this type is still pending (Khruslov 2008). The discovery of a red giant with solar-like oscillations in a long-period eclipsing binary system was recently announced by Hekker et al. (2010).

Semidetached eclipsing binaries with a pulsating primary component which is close to the main sequence were dubbed "oscillating Algols" (oEA, Mkrtichian et al. 2002). About twenty such systems are known to date (Mkrtichian et al. 2007), most of them containing $\delta$ Scuti type components. The first eclipsing binary containing a $\gamma$ Doradus type pulsating star ( VZ CVn) was reported by Ibanoğlu et al. (2007). Recently, Damiani et al. (2010) and Maceroni et al. (2010) identified two $\gamma$ Doradus candidates in eclipsing binaries using CoRoT 
photometry (CoRoT 102931335 and CoRoT 102918586).

CoRoT (Convection, Rotation and planetary Transit, Fridlund et al, 2006) is a space experiment devoted to study the convection and rotation of stars and to detect planetary transits. CoRoT data become publicly available one year after the release to the Co-Is of the mission from the CoRoT archive: http://idoc-corot.ias.u-psud.fr/

In this paper we announce the discovery of a new oEA system identified using publicly available data from the CoRoT initial run (exoplanet field). An almost uninterrupted 55 day long monochromatic CoRoT light curve together with ground-based multicolor photometry allowed us to identify the pulsating component as a likely $\gamma$ Dor type variable, however, the classification as a Slowly Pulsating B (SPB) star cannot be ruled out. The object is an interesting target for a spectroscopic follow-up.

\section{Observational data}

\subsection{CoRoT photometry}

CoRoT 102980178 (USNO-B1.0 0873-0161681, coordinates: $\alpha=06^{\mathrm{h}} 50^{\mathrm{m}} 12^{\mathrm{s}} \cdot 10$, $\delta=-02^{\circ} 41^{\prime} 21^{\prime \prime} .8$, J2000, Monet et al. 2003) was identified by us as an Algol type eclipsing binary with an oscillating component (oEA star) after the visual inspection of the CoRoT light curve. The star was also independently identified as an eclipsing binary by Debosscher et al. (2009) and Carpano et al. (2009). The CoRoT light curve covers almost 55 days from JD 2454138.1 to JD 2454192.8 with $512 \mathrm{sec}$ time sampling. We have removed data points with large error-bars which were typically upward outliers caused by high-energy particles hitting the CCD detector inside the measurement aperture. Most of these events occur when the satellite crosses the South Atlantic Anomaly (SAA). Elimination of these data points introduces small gaps in the light curve, with some of these gaps occurring quasi-periodically (due to SAA crossing). However, the number of discarded data points is relatively small (1168 out of 9229, $12.6 \%)$. To characterize the effect of periodic data rejection on the frequency analysis we have constructed the spectral window plot presented on Fig. 1. The amplitudes of the alias frequencies are of the order of $10 \%$ or below. We have also corrected the light curve for a small $(0.211 \mathrm{mmag} /$ day $)$ downward trend which is probably a result of a gradual drift of the satellite pointing or some kind of instrumental decay. A detailed discussion of noise properties of the CoRoT data can be found in Aigrain et al. (2009) and Auvergne et al. (2009). 
58 New eclipsing binary system with a pulsating component detected by CoRoT

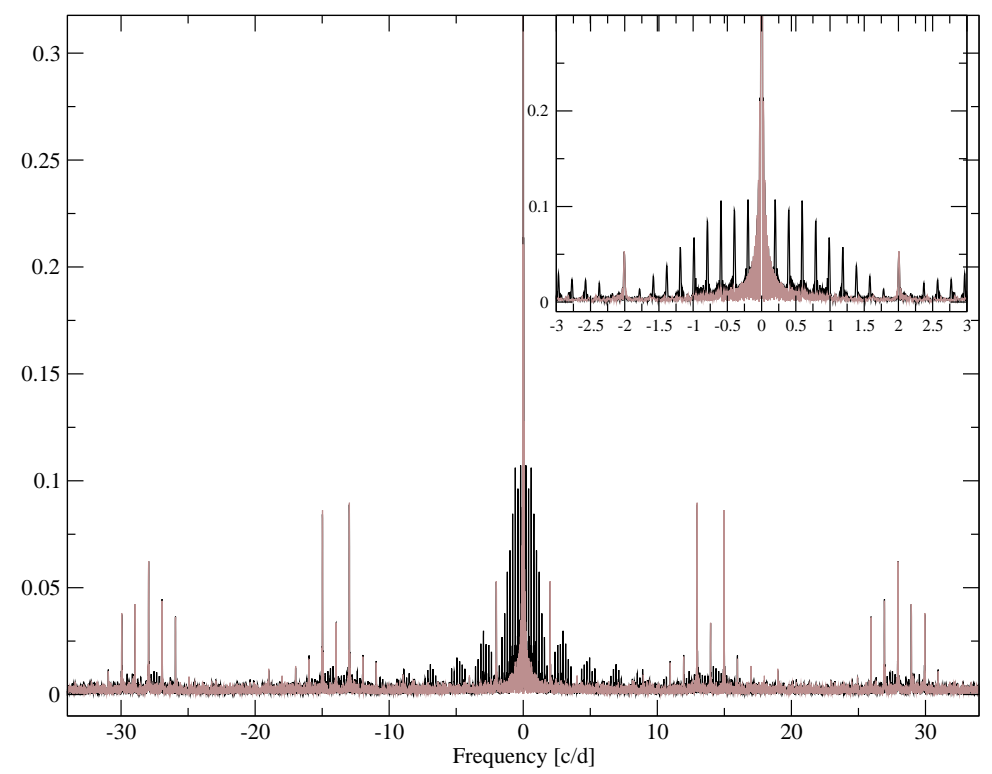

Figure 1: Spectral windows for the outlier-rejected data (light) and for the data excluding the primary eclipses (dark), which were used for frequency analysis. The inset shows a zoom-in to the lower frequencies.

\subsection{Ground-based multicolor data}

In the framework of ground support to the CoRoT mission, we obtained Strömgren uvby photometry of this object using the Wide Field Camera (WFC) on the $2.5 \mathrm{~m}$ Isaac Newton Telescope (INT) at Roque Muchachos Observatory, La Palma, Canary Islands. The observations were conducted on JD 2454170.391 which corresponds to the orbital phase 0.197 of the binary system. The object was also observed in Johnson $B$ and $V$ filters by Deleuil et al. 2006 using the same telescope and camera. Infrared $J H K_{s}$ photometry of this object is available from the 2MASS catalog (Skrutskie et al. 2006). The 2MASS observations were conducted on JD 2451526.704 which corresponds to the orbital phase 0.191 (by a lucky coincidence this is very close to the phase of our Strömgren photometry). The great advantage of the 2MASS photometry is that it was obtained using a camera which utilizes a beam splitter producing truly simultaneous measurements in three filters. Therefore, 2MASS colors are not distorted by stellar variability.

All available color information is summarized in Table 1. 
Table 1: Multicolor photometry of CoRoT 102980178

\begin{tabular}{rrlc} 
Parameter & Value & Error & Origin \\
\hline$b-y=$ & 0.598 & \pm 0.032 & INT/WFC \\
$y=$ & 15.723 & \pm 0.034 & INT/WFC \\
$m_{1}=$ & -0.042 & \pm 0.11 & INT/WFC \\
$c_{1}=$ & 0.891 & \pm 0.086 & INT/WFC \\
$B=$ & 16.58 & \pm 0.45 & INT/WFC \\
$V=$ & 15.73 & \pm 0.19 & INT/WFC \\
$J=$ & 13.411 & \pm 0.028 & 2 MASS \\
$H=$ & 12.759 & \pm 0.023 & 2 MASS \\
$K_{s}=$ & 12.528 & \pm 0.027 & 2 MASS \\
\hline
\end{tabular}

\subsection{Interstellar extinction estimation}

In the absence of spectroscopic data it is important to constrain the influence of interstellar reddening, because information about intrinsic colors is necessary for an unambiguous classification of the pulsating star.

According to the standard tables by Schlegel et al. (1998) the total Galactic extinction in the direction of the object is large: $A_{V}=3$. $860, E(B-V)=$ 1 . 164 . The Schlegel et al. estimation is based on direct observations of farinfrared emission of interstellar dust, material which causes absorption in visible and near-infrared bands. However, as it is noted by the authors, the extinction estimation for this position may be unreliable because of the low Galactic latitude $(b=-1.50)$. There is an alternative way to estimate the Galactic extinction which is based on the relation between $\mathrm{HI}$ column density $\left(N_{H I}\right)$ and extinction caused by dust: $N_{H I} / A_{V}=1.79 \times 10^{21} \mathrm{~cm}^{-2} \mathrm{mag}^{-1}$ (Predehl \& Schmitt 1995). We estimate $N_{H I}$ in the direction of the object using $21 \mathrm{~cm}$ radio observations from the Leiden/Argentine/Bonn Galactic HI Survey (Kalberla et al. 2005, see also http://www . astro. uni-bonn.de/ webaiub/english/ tools_labsurvey.php). From these data we obtain $N_{H I}=0.682 \times 10^{22} \mathrm{~cm}^{-2}$ corresponding to $A_{V}(H I)=3.810$ which is in good agreement with the estimation obtained using the Schlegel et al. tables.

Unfortunately, the distance to the binary system is unknown, as well as the exact distribution of the absorbing material along the line of sight. The intrinsic color of the system lies somewhere between the observed color and the color corrected for the total Galactic reddening along the line of sight. However, even reliable upper limits on the Galactic reddening will be useful for the following discussion. 


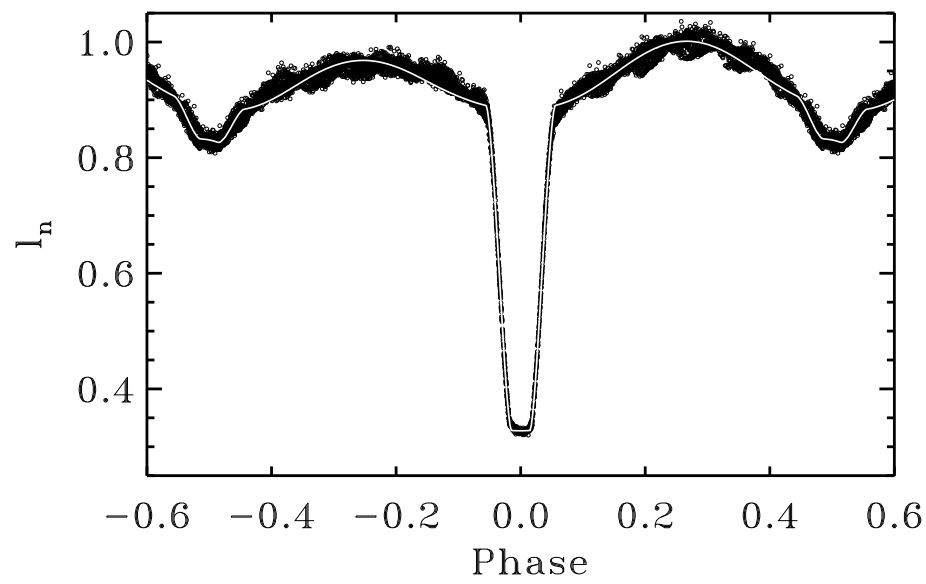

Figure 2: Detrended light curve of CoRoT 102980178 phased with light elements $H J D_{\text {Minl }}=2454139.0680+5^{\mathrm{d}} .0548 \times E$. The solid line represents the F-primary model light curve.

\section{Interpretation and Modeling}

The light curve of CoRoT 102980178 shows eleven 1. 26 deep primary and the equal number of $0 \mathrm{~m} 25$ deep secondary eclipses (at phase 0.50 ). The nearly flat primary minimum bottom indicates that the eclipses are total. Ellipsoidal variability is evident in the out of eclipse light curve (Fig. 2). The maximum preceding the primary minimum is 0 m. 04 fainter than the maximum which follows it (positive O'Connell effect, see Davidge \& Milone 1984 and Liu \& Yang 2003 for a discussion of the effect). The period analysis with the Lafler \& Kinman (1965) method leads to the following light elements:

Individual minima times were estimated using the Kwee \& van Woerden (1956) method and combined to produce a single primary minimum epoch. Its $1 \sigma$ uncertainty was estimated from the scatter of individual measurements on the Observed minus Calculated $(O-C)$ plot produced with the above light elements. Period uncertainty was estimated following Schwarzenberg-Czerny (1991).

Superimposed on this classical Algol-type light curve are 0.01 oscillations with the period of 0 d. 36372 (see Fig. 3 and the detailed discussion below). The oscillations are evident at all phases except during the primary minimum (Fig. 4). 


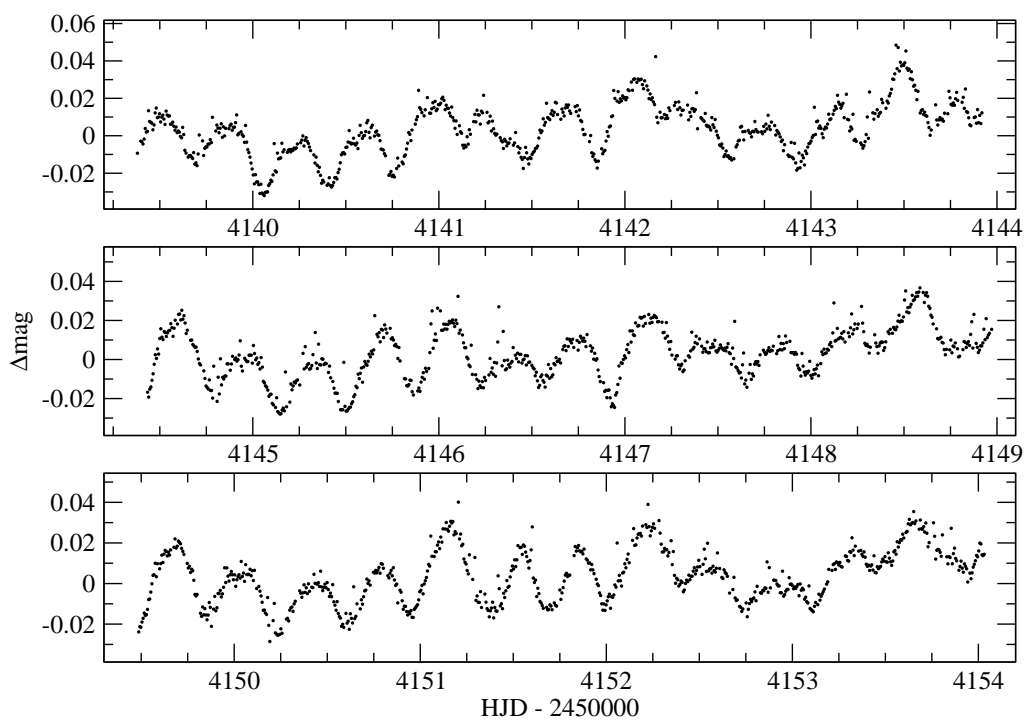

Figure 3: The residual light curve (F-primary model).

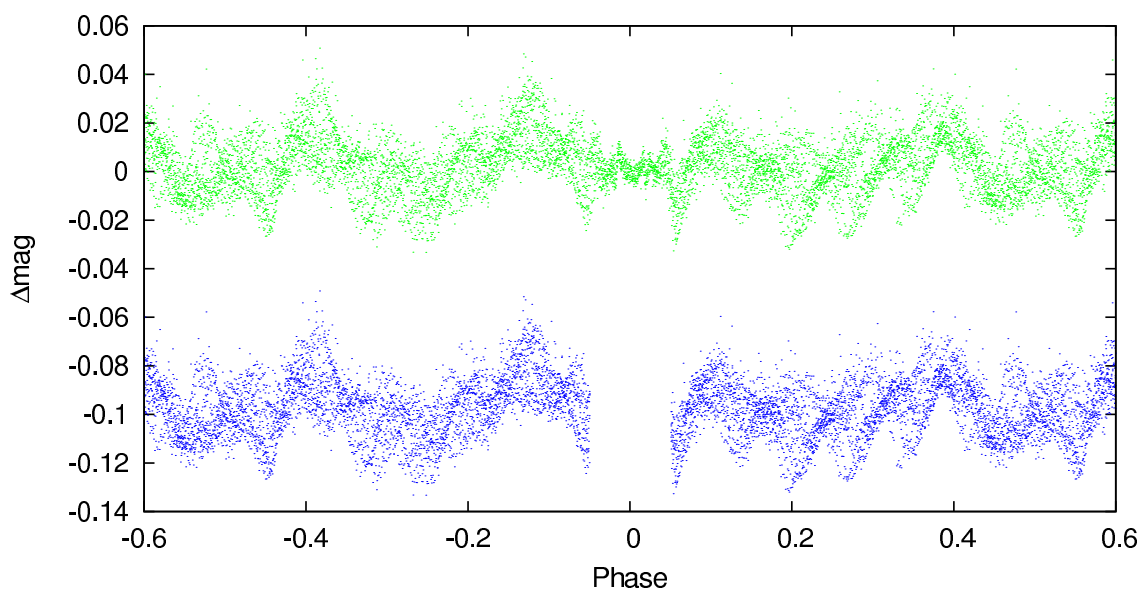

Figure 4: The residual light curve (F-primary model) folded with the binary orbital period. The lower curve represents the section of the residual light curve used for frequency analysis, it was shifted $0 .{ }^{\mathrm{m}} 1$ along the vertical axis for visibility. 


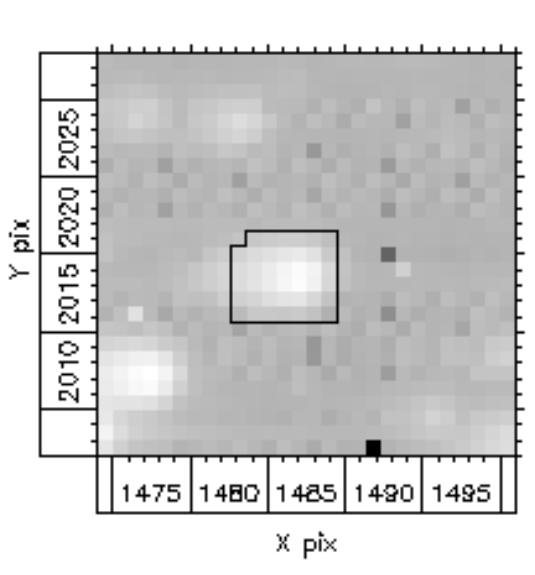

(a) CoRoT image

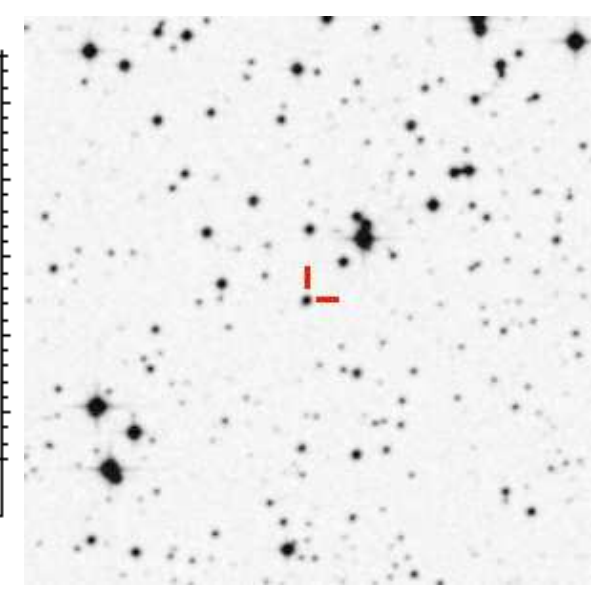

(b) DSS finding chart

Figure 5: (a) CoRoT image of the variable star, the marked area is the measurement aperture. The image is in detector coordinates, east is up, north is to the left. (b) 5' x 5' DSS2 red image centered on CoRoT 102980178 (north is up, east is to the left).

\subsection{Ruling out an optical blend}

When analyzing a variable star showing two different types of variability (e.g. eclipses and pulsations) it is possible that the observed variability comes from two unrelated objects which just happened to be on the same line of sight. For the variable star described here, this possibility may be ruled out due to the following reasons: 1 ) inspections of both the original CoRoT image (Fig. 5a) and the DSS image (Fig. 5b) reveal no other stars in the immediate vicinity of the variable and 2) pulsations disappear during the primary eclipse, which proves that the pulsating star is actually blocked from view.

\subsection{Oscillating component classification}

Following Moon (1986) we calculate reddening-free indices

$$
\begin{gathered}
{\left[c_{1}\right]=c_{1}-0.19(b-y)=0.777 \pm 0.087} \\
{\left[m_{1}\right]=m_{1}+0.33(b-y)=0.155 \pm 0.111}
\end{gathered}
$$

which are consistent with a main sequence star of spectral class B8-9 or A7-F1 within the uncertainty (see Fig. 6). 


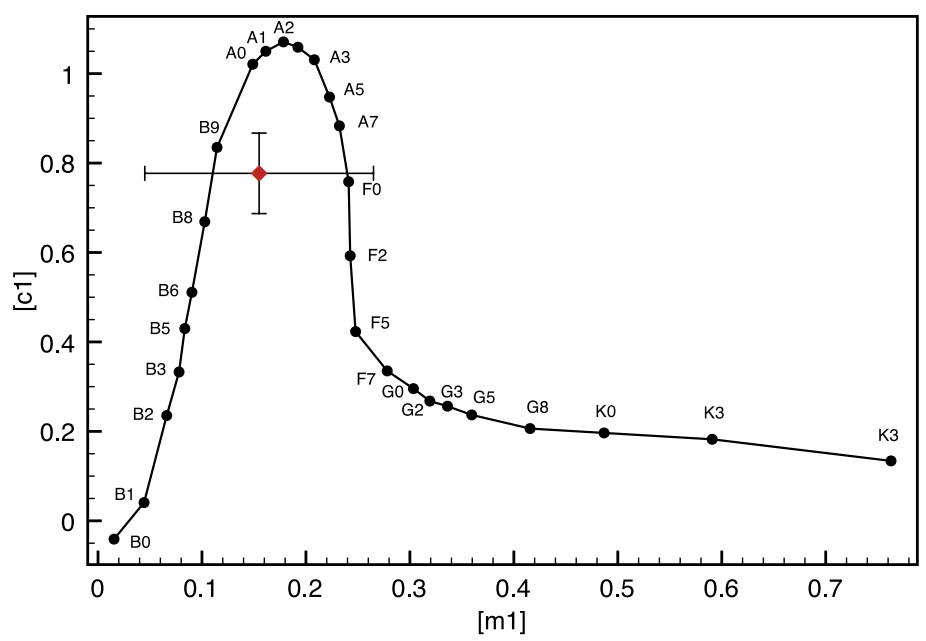

Figure 6: $\left[c_{1}\right]-\left[m_{1}\right]$ plot from Moon (1986) with the position of CoRoT 102980178 and its $1 \sigma$ uncertainty indicated.

The Johnson $B$ and $V$ band observations with INT/WFC by Deleuil et al. (2006) (see Table. 1) have large error bars and where not used in the analysis, however, we note that the $(B-V)$ color of the star does not contradict our proposed spectral classification after accounting for the uncertainties in the Galactic extinction.

2MASS infrared colors can also be used to estimate the spectral class. Accounting for the uncertainty in the Galactic reddening discussed above, the infrared colors correspond to a spectral class in the range of early $A$ - early $M$ (Bessell \& Brett 1988). However, it is possible that the secondary component of the binary system dominates the infrared light, so this spectral type estimation may correspond to the secondary star instead of the pulsating component.

The multicolor photometry excludes an early B spectral class, but is consistent with a reddened late $B$ or $F$ class star.

The period and amplitude of oscillations are typical for $\gamma$ Doradus type but also within the possible range for young Slowly Pulsating B (SPB) and $\beta$ Cephei stars. Information about the spectral class is crucial to distinguish between these possibilities: $\beta$ Cephei variables are early-type B stars (B0 B2.5), SPB stars have spectral types between B2 and B9 (Stankov \& Handler 2005) while $\gamma$ Doradus variables are typically early F type stars (e.g. Rodríguez 2002, Henry et al. 2007). A $\beta$ Cephei classification can be excluded on the basis of multicolor photometry. Note also that according to Pigulski \& Pojmański (2009), $\beta$ Cephei variables are characterized by periods $<0$. 35 while the GCVS 
(Samus et al. 2009) gives 0.6 as the maximum period for this variability class. SPB classification is consistent with the Strömgren photometry, but SPB stars are usually characterized by longer pulsation periods of $0 \mathrm{~d} 5-5^{\mathrm{d}}$ (Thoul 2009) and therefore, we consider the classification of the oscillating component as an SPB star to be less likely. $\gamma$ Doradus stars are characterized by periods ranging from $0 d 4$ to $3^{d}$ (Kaye et al. 1999). Therefore, $\gamma$ Doradus type variable is the most probable classification of the oscillating component.

\subsection{Binary system modeling}

For the construction of the binary system model we have cleaned the light curve from outlier points and corrected it for the linear systematic trend. No prewhitening for the pulsations was done. The oscillations should not affect the binary model because they are significantly smaller than the main features of the eclipsing light curve.

The light curve was analyzed with the PHOEBE software (Prša \& Zwitter 2005, see also http://phoebe.fiz.uni-lj.si), which provides a userfriendly interface and additional capabilities to the Wilson \& Devinney (1971) code. The software was recently modified to take into account CoRoT transmission functions, both for flux and limb darkening computations (for details see Maceroni et al. 2009). Since the orbit-unrelated variability is of much smaller amplitude and shorter period than the eclipses, we just fitted the light curve folded in phase according to the ephemeris presented above (Fig. 2).

After an initial screening of the parameter space, we proceeded to the light curve solution, using - at different stages - both the differential correction and the Nelder and Mead's Simplex algorithm. The fit adjustable parameters were: the inclination, $i$, the mass ratio, $q=M_{2} / M_{1}$, the surface potentials $\Omega_{1,2}$ (which, together with $\mathrm{q}$, determine the components' fractional radii $r_{1,2}$ ), and the secondary effective temperature, $T_{\text {eff }, 2}$. The albedo $a_{i}$ and gravity darkening coefficients $\beta_{i}$ were fixed at the theoretical values $\left(a_{1,2}=1.0,0.5\right.$ and $\beta_{1,2}=1.0,0.32$, respectively, for stars with radiative or convective envelopes). We adopted a non-linear limb darkening law, whose coefficients were interpolated from the PHOEBE tables on-the-fly, according to the star's temperature and surface gravity $(\log g)$. For the F-primary model (see below) we chose a logarithmic limb darkening law, for the B-primary one we chose a square-root law. The primary luminosity in the CoRoT passband, $L_{1}$, was computed at each iteration in PHOEBE, rather than adjusted, to enhance convergence ( $L_{2}$ is derived from the other parameters and model atmospheres).

We started from a detached configuration but in all cases the iterative solution evolved towards a semidetached configuration (with the less massive star in contact). We checked, as well, the effect of non-zero eccentricity, which 
however did not provide any improvement of the fit.

It is well known that the solution of a single passband light curve is mainly sensitive to the $T_{\text {eff }}$ relative values, therefore a shift in $T_{\text {eff, } 1}$ yields a shift in the same direction of $T_{\text {eff,2 }}$. It was therefore possible to fit the light curve incorporating different assumptions about the temperature of the primary (pulsating) component: $T_{\text {eff } 1}=7000 \mathrm{~K}$ (early $\mathrm{F}$ spectral class) and $T_{\text {eff } 1}=11400 \mathrm{~K}$ (late $B$ class). As expected, we obtained different temperatures of the secondary: $T_{\text {eff } 2}=4692$ ( $\mathrm{K}$ class) and $T_{\text {eff } 2}=6162$ (late $F$ ) for the first and second model, respectively. Apart from that, the models are very similar. The uncertainty of $T_{\text {eff }} 2$ is dominated by our poor knowledge of $T_{\text {eff } 1}$. For a particular assumed value of $T_{\text {eff } 1}$ the formal error bars of $T_{\text {eff } 2}$ calculated from the least square fit are less than $10 \mathrm{~K}$.

To achieve an acceptable light curve solution the non-negligible O'Connell effect shall also be modeled, and for this purpose we introduced a bright spot on the primary component. Its parameters (location, size and temperature contrast factor, $T_{\text {spot } 1} / T_{\text {eff } 1}$ ) were not adjusted, but determined by trial and error, by putting the spot on the stellar equator and varying only the spot longitude and contrast factor (as the size and the latitude are highly correlated with them, see for example the discussion by Maceroni \& van't Veer 1993). It is reasonable to expect the existence of such a spot as the result of mass accretion from the secondary component. However, different solutions (such as a dark spot on the colder secondary star, in this case due to surface activity), cannot be ruled out. The model parameters are summarized in Table 2.

Table 2: Model parameters of the binary system

\begin{tabular}{rcc} 
Parameter & Model with F primary & Model with B primary \\
\hline assumed $T_{\text {eff } 1}$ & $7000 \mathrm{~K}$ & $11400 \mathrm{~K}$ \\
$T_{\text {eff } 2}$ & $4692 \mathrm{~K}$ & $6162 \mathrm{~K}$ \\
$q=M_{2} / M_{1}$ & $0.206 \pm 0.001$ & $0.184 \pm 0.001$ \\
$i$ & $84^{\circ} .61 \pm 0.05$ & $85^{\circ} .61 \pm 0.04$ \\
$r_{1}$ & $0.1068 \pm 0.0002$ & $0.1064 \pm 0.0002$ \\
$r_{2}$ & $0.2447 \pm 0.0003$ & $0.2363 \pm 0.0003$ \\
$L_{2} / L_{1}$ & $0.7070 \pm 0.0005$ & $0.7183 \pm 0.005$ \\
$T_{\text {spot } 1} / T_{\text {eff } 1}$ & 1.22 & 1.50 \\
\hline
\end{tabular}

The spot angular radius was fixed to $15^{\circ}$, its colatitude to 90 , the longitude of $240^{\circ}$ and the temperature contrast factors in the table were found by trial and error. The uncertainties are formal fit errors $(1 \sigma)$.

One of the light curve features which are not reproduced by the models is a small difference in depth among primary eclipses. The eleven observed eclipses 
are not sufficient to draw a conclusion about a possible periodicity of the primary eclipse depth variation. The models also predict a symmetric light curve shape during the total eclipse phase which is not observed. These two effects may be caused by starspots on the secondary component. As can be seen from Fig. 2, there are some residual systematic deviations of the model with respect to observations. This will imply the presence of the orbital frequency $\left(f_{\text {orb }}\right)$ and its multiples (typically the even ones) in the residual light curve spectrum which is discussed in the following section.

\subsection{Pulsation frequency analysis}

The residual light curve resulting from the subtraction of the binary model (version with early-F type primary) is presented in Fig. 3. It shows clear pulsation with a dominant frequency of $\sim 2.75 \mathrm{c} / \mathrm{d}$. If folded with the binary orbital period (see Fig. 4) the residual light curve shows a rather complicated pattern instead of uncorrelated data. This means that the main pulsation frequency is close to an integer multiple of the binary orbital frequency $\left(\mathrm{f} 1 / \mathrm{f}_{\text {orb }}=13.898 \sim 14\right)$. The pulsation disappears during the primary eclipse (phases $-0.05-0.05$ on Fig. 4), and this phase interval was excluded from the frequency analysis. In order to check for possible artifacts introduced due to omitting parts of the light curve, the analysis was repeated including observations during total eclipses. The introduced artifacts were found to be negligible.

The frequency analysis was performed using the SigSpec software (Reegen 2007), leading to 99 formally significant frequencies including the CoRoT orbit and its harmonics. The first few frequencies are listed in Table 3. The search for combination frequencies was performed using the software COMBINE developed by P. Reegen (see http://www.SigSpec.org). The frequency errors were estimated following Kallinger et al. (2008). The orbital frequency of the binary system was added to the frequency list, because from the plot of the resulting frequencies (Fig. 7) it is obvious that there are many combinations with the orbital frequency.

Only two frequencies ( $\mathrm{f} 1$ and $\mathrm{f} 3$ in Table 3 ) appear to be genuine, which means not explained by a combination; $f 3$ is close to $f_{\text {orb }}$ but the frequency errors are small enough to conclude that the frequencies are significantly different (at $10 \sigma$ level).

Analysis of the residual light curve after the subtraction of the B primary model leads to similar results (Table 3 ). The one major difference is that f8 becomes the second most powerful harmonic in the spectrum. Its amplitude is nine times larger compared to residuals from the binary model with an early- $F$ type primary. $\mathrm{f} 8$ in the $\mathrm{B}$ primary model residual spectrum can be associated with the orbital frequency $\left(f_{\text {orb }}=0.1978 \pm 0.0007\right)$, the difference between them 
Table 3: Frequencies detected in the residual light curve

\begin{tabular}{rcc|cc} 
& \multicolumn{2}{c}{ Model with F primary } & \multicolumn{2}{c}{ Model with B primary } \\
& $\begin{array}{c}\text { Frequency } \\
{[\mathrm{c} / \mathrm{d}]}\end{array}$ & $\begin{array}{c}\text { Amplitude } \\
{[\mathrm{mmag}]}\end{array}$ & $\begin{array}{c}\text { Frequency } \\
{[\mathrm{c} / \mathrm{d}]}\end{array}$ & $\begin{array}{c}\text { Amplitude } \\
{[\mathrm{mmag}]}\end{array}$ \\
\hline $\mathrm{f} 1:$ & $2.7494 \pm 0.0008$ & $9.52 \pm 0.42$ & $2.7494 \pm 0.0009$ & $9.68 \pm 0.48$ \\
$\left(4 \mathrm{f}_{\text {orb }}\right) \mathrm{f} 2:$ & $0.7917 \pm 0.0008$ & $7.48 \pm 0.35$ & $0.7920 \pm 0.0009$ & $6.31 \pm 0.32$ \\
$\mathrm{f} 3:$ & $0.2115 \pm 0.0012$ & $3.28 \pm 0.25$ & $0.2181 \pm 0.0021$ & $1.97 \pm 0.23$ \\
$\left(2 \mathrm{f}_{\text {orb }}\right) \mathrm{f} 4:$ & $0.4004 \pm 0.0015$ & $3.19 \pm 0.26$ & $0.4012 \pm 0.0016$ & $2.57 \pm 0.22$ \\
$\left(10 f_{\text {orb }}\right) \mathrm{f} 5:$ & $1.9784 \pm 0.0017$ & $3.27 \pm 0.30$ & $1.9783 \pm 0.0014$ & $3.86 \pm 0.30$ \\
$\left(8 f_{\text {orb }}\right) \mathrm{f} 6:$ & $1.5823 \pm 0.0017$ & $2.81 \pm 0.27$ & $1.5822 \pm 0.0013$ & $4.17 \pm 0.29$ \\
$\left(13 f_{\text {orb }}\right) \mathrm{f} 7:$ & $2.5657 \pm 0.0019$ & $2.48 \pm 0.25$ & $2.5650 \pm 0.0019$ & $2.32 \pm 0.25$ \\
$\left(\mathrm{f}_{\text {orb }}\right) \mathrm{f} 8:$ & $0.1978 \pm 0.0031$ & $1.00 \pm 0.17$ & $0.1940 \pm 0.0008$ & $9.05 \pm 0.40$ \\
& $\ldots$ & & $\ldots$ &
\end{tabular}

is less than $4 \sigma$. Most likely, it is not a real frequency but an artifact introduced by an imperfect binary model subtraction.

All the other frequencies can be explained by combinations mainly with the orbital frequency. This is what can be expected from a close binary because the primary component is likely to be distorted by tidal forces. If the $F$ type primary hypothesis is accepted, the solution for the independent frequencies has two components: f1 and f3.

\section{Conclusions}

We have identified CoRoT 102980178 as a semidetached eclipsing binary with a 5.0548 orbital period. It shows an Algol-type light curve with 1. 26 deep primary and 0 . 25 deep secondary eclipses (in CoRoT photometric band).

Our light curve modeling leads to a semidetached configuration with the photometric mass ratio $q=0.2$ and orbital inclination $i=85^{\circ}$. We note that the inverse problem of binary model fitting using only a broad passband light curve is degenerate and the proposed solution might not be unique.

The more massive component of the system is pulsating with the primary frequency of $2.7494 \mathrm{c} / \mathrm{d}(0 \mathrm{~d} 36372$ period) and 0.01 amplitude. The observed period and amplitude of pulsations as well as ground-based multicolor photometry favor its classification as a $\gamma$ Doradus type variable, however, based on our data we cannot exclude the SPB-hypothesis for the primary. The detailed frequency analysis suggests the presence of an additional independent pulsation mode with the frequency of $0.21-0.22 \mathrm{c} / \mathrm{d}$. Other detected frequencies can be explained as combinations of the above frequencies and the orbital frequency. 


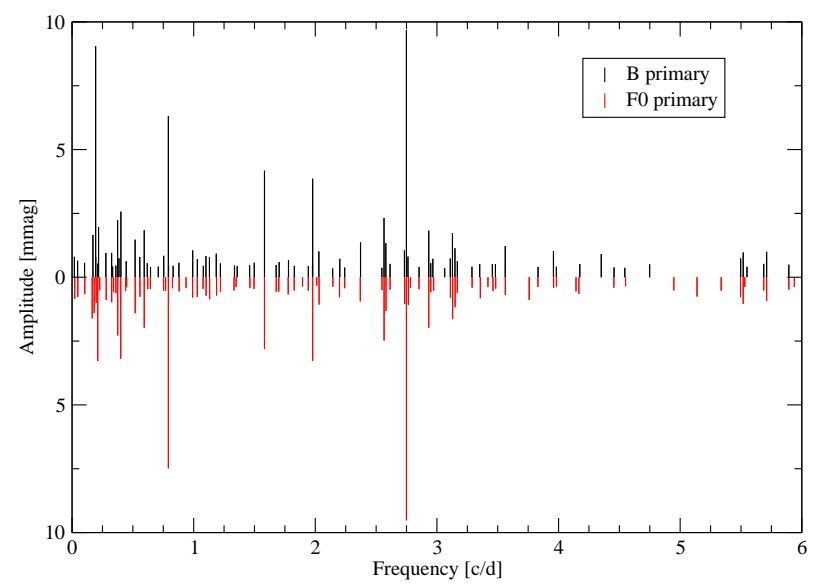

Figure 7: Amplitude spectrum of the residual light curve after subtracting models with B (black) and F (red, plotted downwards for better visibility) primary.

The system deserves a detailed spectroscopic study to better constrain physical parameters of the pulsating component. A Strömgren photometry of the primary eclipse could also be useful for this purpose.

Acknowledgments. Based on observations made with the WFC at the INT operated on the island of La Palma by the Isaac Newton Group in the Spanish Observatorio del Roque de los Muchachos of the Instituto de Astrofisica de Canarias. We would like to thank Sergei Antipin and Nikolai Samus for their aid in the classification of this peculiar object, Suzanne Aigrain and Werner Weiss for their help in establishing this fruitful collaboration, as well as Nicola Marchili, Frank Schinzel and the anonymous referee for reviewing this manuscript. KS was supported through a stipend from the International Max Planck Research School (IMPRS) for Astronomy and Astrophysics at the Universities of Bonn and Cologne. $\mathrm{CM}$ and $\mathrm{CD}$ research has been funded by the Italian Space Agency (ASI) under contract ASI/INAF I/015/07/00 in the frame of the ASIESS project. This research has made use of the Exo-Dat database, operated at LAM-OAMP, Marseille, France, on behalf of the CoRoT/Exoplanet program. The publication makes use of data products from the Two Micron All Sky Survey, which is a joint project of the UMass/IPAC-Caltech, funded by the NASA and the NSF, the Aladin interactive sky atlas, operated at CDS, Strasbourg, France, the International Variable Star Index (VSX) operated by the AAVSO and the NASA/IPAC Extragalactic Database (NED) which is operated by the 
JPL, Caltech, under contract with the NASA. This research has made use of NASA's Astrophysics Data System.

\section{References}

Aigrain, S., Pont, F., Fressin, F., et al. 2009, A\&A, 506, 425

Antipin, S. V., Sokolovsky, K. V., \& Ignatieva, T. I. 2007, MNRAS, 379L, 60

Araujo-Betancor, S., Gänsicke, B. T., Hagen, H.-J., et al. 2005, A\&A, 430, 629

Auvergne, M., Bodin, P., Boisnard, L., et al. 2009, A\&A, 506, 411

Bessell, M. S., \& Brett, J. M. 1988 PASP, 100, 1134

Bíró, I. B., \& Nuspl, J. 2005, ASPC, 333, 221

Carpano, S., Cabrera, J., Alonso, R., et al., 2009, A\&A, 506, 491

Chochol, D., \& Pribulla, T. 2000, ASPC, 203, 125

Davidge, T. J., \& Milone, E. F. 1984, ApJS, 55, 571

Damiani, C., Maceroni, C., Cardini, D., et al. 2010, Ap\&SS, 66

Debosscher, J., Sarro, L. M., López, M., et al., 2009, A\&A, 506, 519

Deleuil, M., Moutou, C., Deeg, H. J., et al. 2006, ESA Special Publication, 1306, 341

Fridlund, M., Baglin, A., Lochard, J., \& Conroy, L. 2006, ESASP, 1306,

Handler, G., Balona, L. A., Shobbrook, R. R., et al. 2002, MNRAS, 333, 262

Hekker, S., Debosscher, J., Huber, D., et al. 2010, ApJ, 713, L187

Henry, G. W., Fekel, F. C., \& Henry, S. M. 2007 AJ, 133, 1421

Ibanoğlu, C., Taș, G., Sipahi, E., \& Evren, S. 2007, MNRAS, 376, 573

Kalberla, P. M. W., Burton, W. B., Hartmann, D., et al. 2005, A\&A, 440, 775

Kallinger, T., Reegen, P., \& Weiss, W. W. 2008, A\&A, 481, 571

Kaye, A. B., Handler, G., Krisciunas, K., Poretti, E., \& Zerbi, F. M. 1999, PASP, 111,840

Khruslov, A. V. 2008, PZ, 28, 4

Kwee, K. K., \& van Woerden, H. 1956, BAN, 12, 327

Lafler, J., \& Kinman, T. D. 1965, ApJS, 11, 216

Liu, Q.-Y., \& Yang, Y.-L. 2003, ChJAA, 3, 142

Maceroni, C., Montalbán, J., Michel, E., et al. 2009, A\&A, 508, 1375

Maceroni, C. et al. 2010 in preparation; to appear in AN

Mkrtichian, D. E., Kusakin, A. V., Gamarova, A. Y., \& Nazarenko, V. 2002, ASPC, 259, 96

Mkrtichian, D. E., Kim, S.-L., Rodríguez, E., et al. 2007, ASPC, 370, 194

Monet, D. G., Levine, S. E., Canzian, B., et al. 2003, AJ, 125, 984 
70 New eclipsing binary system with a pulsating component detected by CoRoT

Moon, T. 1986, Ap\&SS, 122, 173

Pigulski, A., \& Pojmański, G. 2009, AIPC, 1170, 351

Predehl, P., \& Schmitt, J. H. M. M. 1995, A\&A, 293, 889

Prša, A., \& Zwitter, T. 2005, ApJ, 628, 426

Reegen, P. 2007, A\&A, 467, 1353

Rodríguez, E. 2002 ESASP, 485, 331

Samus, N. N., Durlevich, O. V., et al. 2009, yCat, 1, 2025

Schlegel, D. J., Finkbeiner, D. P., \& Davis, M. 1998, ApJ, 500, 525

Schwarzenberg-Czerny, A. 1991 MNRAS, 253, 198

Skrutskie, M. F., Cutri, R. M., Stiening, R., et al. 2006, AJ, 131, 1163

Stankov, A., \& Handler, G. 2005, ApJS, 158, 193

Thoul, A. 2009, CoAst, 159, 35

Wilson, R. E., \& Devinney, E. J. 1971, ApJ, 166, 605 